\title{
Role of Alix in miRNA packaging during extracellular vesicle biogenesis
}

\author{
ALESSANDRA IAVELLO ${ }^{1}$, VALESKA S.L. FRECH ${ }^{1}$, CHIARA GAI ${ }^{1}$, \\ MARIA CHIARA DEREGIBUS ${ }^{1}$, PETER J. QUESENBERRY ${ }^{2}$ and GIOVANNI CAMUSSI ${ }^{1}$ \\ ${ }^{1}$ Department of Medical Sciences and Molecular Biotechnology Center, University of Torino, 10126 Torino, Italy; \\ ${ }^{2}$ Department of Medicine, The Warren Alpert Medical School of Brown University, Providence, RI 02903, USA
}

Received October 20, 2015; Accepted January 21, 2016

DOI: $10.3892 / \mathrm{ijmm} .2016 .2488$

\begin{abstract}
Evidence indicates that Alix, an accessory protein of the endosomal sorting complex required for transport (ESCRT), is involved in the biogenesis of extracellular vesicles (EVs). EVs contain selected patterns of microRNAs (miRNAs or miRs); however, little is known about the mechanisms of miRNA enrichment in EVs. The aim of the present study was to evaluate whether Alix is involved in the packaging of miRNAs within EVs released by human liver stem-like cells (HLSCs). EVs released from HLSCs were enriched with miRNAs and expressed Alix and several RNA-binding proteins, including Argonaute 2 (Ago2), a member of the Argonaute family known to be involved in the transport and the processing of miRNAs. Co-immunoprecipitation experiments revealed an association between Alix and Ago2. The results from RT-qPCR indicated that in the Alix/Ago2 immunoprecipitates, miRNAs were detectable. EVs were instrumental in transferring selected miRNAs from HLSCs to human endothelial cells absent in the latter cells. Alix knockdown did not influence the number of EVs released by HLSCs, but it significantly decreased miRNA expression levels in the EVs and consequently their transfer to the endothelium. Our findings indicate that Alix binds to Ago2 and miRNAs, suggesting that it plays a key role in miRNA enrichment during EV biogenesis. These results may represent a novel function of Alix, demonstrating its involvement in the EV-mediated transfer of miRNAs.
\end{abstract}

Correspondence to: Professor Giovanni Camussi, Dipartimento di Scienze Mediche, Corso Dogliotti 14, 10126 Torino, Italy

E-mail: giovanni.camussi@unito.it

Abbreviations: EVs, extracellular vesicles; HLSCs, human liver stem-like cells; HUVECs, human umbilical cord vein-derived endothelial cells; ESCRT, endosomal sorting complex required for transport; Ago2, argonaute 2; miRNAs, microRNAs; Stau 1, Staufen 1; Stau 2, Staufen 2; TIA-1, T-cell internal antigen-1; TIAR, TIA-1-related protein; HuR, human antigent R; Co-IP, co-immunoprecipitation; RT-qPCR, reverse transcriptase-quantitative polymerase chain reaction; Rq, relative quantification; ActD, actinomycin $\mathrm{D}$

Key words: exosomes, microvesicles, Alix, Argonaute 2, microRNAs

\section{Introduction}

It has been recognized that under physiological and pathological conditions, cells may release vesicles that may be instrumental in intercellular communication as they carry biologically active molecules (1-4). The non-apoptotic vesicles have been classified based on their biogenesis into exosomes derived from membranes of the endosomal compartment and microvesicles that are formed from the budding of a portion of the cell plasma membrane $(5,6)$. Given the heterogeneity of the released vesicles and the overlapping features of molecules carried by exosomes and microvesicles, it has been suggested that they be collectively termed extracellular vesicles (EVs) (7). During vesiculation, nucleic acids, including mRNAs, long non-coding RNAs (lncRNAs) and microRNAs (miRNAs or miRs) are encapsulated within EVs and when released, they are protected from degrading enzymes (8-10). Therefore, EVs may carry genetic information that may be shared among cells (11-14). Aliotta et al (15) demonstrated that lung-derived vesicles carried RNA to marrow cells and altered the phenotype of these cells both genetically and functionally. The horizontal transfer of extracellular RNAs carried by EVs has been shown to be able to reprogram hematopoietic progenitors (11) and to activate endothelial cells (12). The EV-mediated transfer of miRNAs is a particularly good candidate for the epigenetic alterations observed in recipient cells. In fact, it has been shown that EVs are enriched in miRNAs and that they can be transferred to target cells $(13,14)$. Gibbings et al $(16)$ suggested that the constituents of the miRNA effector complex, such as GW182 and Argonaute 2 (Ago2), are involved in the packaging of miRNAs within multivesicular bodies during exosome biogenesis. We previously found that EVs derived from adult stem cells contain several ribonucloproteins (14) known to be implicated in RNA storage and/or stability, such as Staufen 1 and 2 (Stau1/2), T-cell internal antigen-1 (TIA-1), TIA-1-related protein (TIAR), the AU-rich element binding protein, human antigent R (HuR), and Ago2 (17,18), which is involved in the transport and processing of miRNAs (19). Vesicles derived from adult human liver stem-like cells (HLSCs) have also been shown to contain ribonucleoproteins and different RNA species involved in hepatic regeneration (20). The efficacy of HLSCs in restoring hepatic mass and liver functionality has been shown $(20,21)$. HLSCs do not express hematopoietic 
stem cell markers, whereas they express several mesenchymal markers and display multiple differentiating abilities (22). Moreover, HLSCs express stem cells and embryonic markers, including alpha-fetoprotein (AFP), octamer-binding transcription factor 3/4 (Oct 3/4), Sox2, Musashil, nestin, nanog and pax2 $(20,22)$. The expression of albumin and AFP suggests a partial hepatic commitment $(20,22)$.

Moreover, we found that HLSC-derived EVs were enriched and transferred to tumor cells through antitumor miRNAs that induced tumor regression and inhibited vascularization both in vitro and in vivo (23). However, little is known about the mechanisms of miRNA enrichment in HLSC-derived vesicles. Several studies have indicated that Alix, an accessory protein of the endosomal sorting complex required for transport (ESCRT), is involved in the biogenesis of EVs (24-27) and has multifunctional activities due to its domains, which provide multiple protein-binding sites (24,28-33).

The aim of the present study was to evaluate whether Alix is involved in the packaging of miRNAs within EVs released by HLSCs. For this purpose, co-immunoprecipitation (Co-IP) experiments of Alix with Ago2 were performed and the presence of selected miRNA immunoprecipitates was investigated. Furthermore, Alix was knocked down in HLSCs in order to investigate its role in miRNA enrichment within released EVs.

\section{Materials and methods}

Cell cultures. The HLSCs were obtained from normal human cryopreserved hepatocytes (Lonza, Basel, Switzerland) and their culture and characterization have been previously described $(20,22)$. Briefly, the HLSCs were cultured in medium composed of 3 to 1 proportion of $\alpha$-minimum essential medium ( $\alpha$-MEM) and endothelial cell basal medium (EBM)-1 (both from Lonza, Verviers, Belgium), supplemented with $10 \%$ EV-free fetal bovine serum (FBS; Sigma-Aldrich, St. Louis, MO, USA), $2 \mathrm{mM}$ L-glutamine and $100 \mathrm{IU} / \mathrm{ml}$ penicillin and $100 \mu \mathrm{g} / \mathrm{ml}$ streptomycin. The HLSCs were used at approximately $80 \%$ confluence and at passages 3 to 7 . The HLSCs were characterized for the expression of the mesenchymal stem cell (MSC) markers, CD29, CD44, CD73 and CD90, several embryonic markers, such as Nanog, Nestin, Oct 3/4, Sox2, Musashi1, AFP and Pax2, and the hepatic marker, albumin, as previously described $(20,22)$. Human umbilical cord vein-derived endothelial cells (HUVECs) were cultured in EBM supplemented with the EGM kit (Lonza) (12).

Isolation and characterization of HLSC-derived EVs. EVs were obtained from supernatants of the HLSCs cultured in RPMI serum-free medium supplemented with $100 \mu \mathrm{g} / \mathrm{ml}$ penicillin and $100 \mu \mathrm{g} / \mathrm{ml}$ streptomycin for $16 \mathrm{~h}$. Following centrifugation at $3 \mathrm{~kg}$ for $20 \mathrm{~min}$ to remove debris, cell-free supernatants were centrifuged at $10 \mathrm{~kg}$ for $1 \mathrm{~h}$ at $4^{\circ} \mathrm{C}$ to remove large vesicles, and the supernatant recovered was submitted to a second ultracentrifugation at $100 \mathrm{~kg}$ for $3 \mathrm{~h}$ at $4^{\circ} \mathrm{C}$ (Beckman Coulter Optima L-90K ultracentrifuge; Beckman Coulter, Fullerton, CA, USA). The protein content was quantified by the Bradford method (Bio-Rad, Hercules, CA, USA) and the EV profile was analyzed and counted by NanoSight (NanoSight Ltd., Minton Park, UK) equipped with nanoparticle tracking analysis (NTA) 2.3 analytic software (34).
Flow cytometric analyses of typical HLSC markers were performed on the EVs as previously described (35). In selected experiments, the EVs were labeled with PKH26 dye (Sigma-Aldrich), a red fluorescent aliphatic chromophore $(12,23)$, and with green fluorescent $\mathrm{SYTO}^{\circledR}$ RNASelect (Molecular Probes, Inc., Eugene, OR, USA) (23). After labeling, the EVs were washed by ultracentrifugation at $100 \mathrm{~kg}$ for $1 \mathrm{~h}$ at $4^{\circ} \mathrm{C}$ and re-suspended in RPMI serum-free medium. To prove that the labeled EVs were membrane vesicles and not aggregated proteins, the vesicles were treated with $10 \%$ Triton X-100 (Bio-Rad).

As observed under a confocal microscope (Zeiss LSM 5 Pascal; serial no. 1206000125; Carl Zeiss, Oberkochen, Germany), the EVs were stained with PKH26, which labeled membrane phospholipids, indicating that particles detected in the $100 \mathrm{~kg}$ ultracentrifugation fractions were not protein aggregates. This was confirmed by treatment with Triton $\mathrm{X}$ that was capable of solubilizing the vesicles, but not protein aggregates. Moreover, the PKH26-labeled EVs co-stained with SYTO-RNA dye indicated the presence of RNA within vesicles as previously described (14).

To evaluate EV internalization in the HUVECs, the PKH26-labeled EVs were incubated at $37^{\circ} \mathrm{C}$ for different periods of time and after cell washing the internalization was evaluated by confocal microscopy and fluorescence-activated cell sorting (FACS) analysis, as previously described (23).

Transmission electron microscopy was performed on the EVs derived from the HLSCs suspended in phosphate buffered saline (PBS) and loaded onto 200 mesh nickel Formvar carboncoated grids (Electron Microscopy Science, Hatfield, PA, USA) for 20 min. Following fixation with $2.5 \%$ glutaraldehyde containing $2 \%$ sucrose and repeated washings, the samples were negatively stained with NanoVan (Nanoprobes, Yaphank, NY, USA) and observed using a JEM-1010 electron microscope (Jeol, Tokyo, Japan).

Immunogold electron microscopy was performed as previously described (14). Briefly, the EVs derived from the HLSCs suspended in PBS were loaded onto 200 mesh nickel Formvar carbon-coated grids. After 15 min of fixation in $3 \%$ paraformaldheyde (PAF) in PBS containing 2\% sucrose, the EVs were permeabilized with cold methanol $\left(-20^{\circ} \mathrm{C}\right)$ for 10 min, extensively washed and blocked with $1 \%$ bovine serum albumin (BSA) in PBS (pH 7.4). The EVs were then immunolabeled with Ago2 primary antibody (Cat. no. ab57113; Abcam, Cambridge Science Park, Cambridge, UK) for $1 \mathrm{~h}$ at room temperature. As a control, the primary antibody was omitted. After washing with $1 \%$ BSA in PBS (pH 8.2), the EVs were then incubated with EM goat anti-mouse $\operatorname{IgG}(\mathrm{H}+\mathrm{L})$ $5 \mathrm{~nm}$ Immunogold conjugate antibody (Cat. no. EM.GMHL5; British Biocell International, Cardiff, UK) for $1 \mathrm{~h}$ in PBS/ BSA ( $\mathrm{pH}$ 8.2) at room temperature. The EVs were post-fixed in $2.5 \%$ gluteraldehyde, washed and embedded in a mixture of uranyl acetate $(0.8 \%)$ and methyl cellulose $(0.13 \%)$ following silver enhancement (British Biocell International). The samples were observed under a Jeol JEM-1010 electron microscope.

Western blot analysis. The lysis of cells or EVs was performed in lysis buffer [50 mmol/1Tris-HCl(pH8.3), $1 \%$ TritonX-100,10 mol/1 phenylmethylsulfonyl fluoride (PMSF), $10 \mathrm{~mol} / \mathrm{l}$ leupeptin and $100 \mathrm{U} / \mathrm{ml}$ aprotinin] for $1 \mathrm{~h}$ at $4^{\circ} \mathrm{C}$. Following centrifuga- 
tion at $14,000 \mathrm{x} \mathrm{g}$, the protein content in the supernatant was measured using the Bradford assay. Protein lysates $(50 \mu \mathrm{g})$ were subjected, under reducing conditions, to $10 \%$ sodium dodecyl sulfate-polyacrylamide gel electrophoresis and transferred onto filters of nitrocellulose membranes. The membranes were incubated with $5 \%$ non-fat milk in $20 \mathrm{mmol} / \mathrm{l}$ Tris- $\mathrm{HCl}(\mathrm{pH} 7.5)$, $500 \mathrm{mmol} / 1 \mathrm{NaCl}$ plus $0.1 \%$ Tween (TBS-T) and immunoblotted at $4^{\circ} \mathrm{C}$ for $12 \mathrm{~h}$ with the appropriate concentration of relevant primary antibodies: hepatocyte growth factor-regulated tyrosine kinase substrate (Hrs; mouse monoclonal IgG; Cat. no. sc-101280), tumor susceptibility gene (Tsg)101 (mouse monoclonal IgG; Cat. no. sc-101254), charged multivesicular body protein (CHMP)4a (goat polyclonal IgG; Cat. no. sc-49913), CHMP4b (rabbit polyclonal IgG; Cat. no. sc-82556), CHMP4c (goat polyclonal IgG; Cat. no. sc-82560), Alix (mouse monoclonal IgG; Cat. no. sc-271975), TIA-1 (goat polyclonal IgG; Cat. no. sc-1751), TIAR (goat polyclonal IgG; Cat. no. sc-1749), HuR (mouse monoclonal IgG; Cat. no. sc-5261), Stau1 (mouse monoclonal IgG; Cat. no. sc-376123), Stau2 (mouse monoclonal IgG; Cat. no. sc-101144), STAM (mouse monoclonal IgG; Cat. no. sc-133092) (Santa Cruz Biotechnology, Santa Cruz, CA, USA), anti-ribosomal protein S29 (RPS29; rabbit polyclonal IgG; Cat. no. ab56224; Abcam) Ago2 (mouse monoclonal IgG; Cat. no. ab57113; Abcam), CD63 and actin (Santa Cruz Biotechnology) for normalization. The membranes were extensively washed in TBS-T and incubated with peroxidase-conjugated isotype-specific secondary antibodies [goat anti-rabbit IgG (Cat. no. 31462); goat anti-mouse IgG (Cat. no. 31432); mouse anti-goat IgG (Cat. no. 31400); Thermo Scientific Pierce, Rockford, IL, USA] for $1 \mathrm{~h}$ at room temperature. After washing with TBS-T, the reaction was revealed using ECL detection reagents (Clarity Western ECL Substrate, Bio-Rad) in a ChemiDoc ${ }^{\mathrm{TM}} \mathrm{XRS}^{+}$system (Bio-Rad).

Co-IP assays on the cells and the EVs were performed with Protein A/G PLUS-Agarose beads (Santa Cruz Biotechnology). Briefly, the cells or EV preparations were lysed as previously described (36). Pre-clear lysate (100-500 $\mu \mathrm{g}$ total protein), according to the manufacturer's instructions, were incubated in the presence of the primary mouse antibodies $(2 \mu \mathrm{g})$, Ago 2 or Alix for $1 \mathrm{~h}$ at $4^{\circ} \mathrm{C}$. To the mixture, $20 \mu \mathrm{l}$ of re-suspended volume of Protein A/G PLUS-Agarose was added and incubated at $4^{\circ} \mathrm{C}$ on a rocker platform overnight. Bound proteins were collected by centrifugation at 2,500 rpm and washed 4 times with $1.0 \mathrm{ml}$ RIPA. After a final wash, the pellets were re-suspended in boiling Laemmli buffer. Re-suspended proteins were then subjected to western blot analysis as described above.

Co-IP/protein-RNA interactions. The cell or EV preparations were lysed with lysis buffer [20 mM Tris- $\mathrm{HCl}, 150 \mathrm{mM}$ $\mathrm{NaCl}, 0.5 \%$ Nonidet P-40, 2 mM EDTA, $0.5 \mathrm{mM}$ dithiothreitol (DTT), $1 \mathrm{mM} \mathrm{NaF}, 1 \mathrm{mM}$ PMSF, $1 \%$ protease inhibitor cocktail from Sigma (pH 7.5) and RNase inhibitor (800 U/ml)]. The immunoprecipitation assay was performed as previously described (36). To evidence the background RNA contamination, a normal mouse antibody was used. After a final wash, the pellets were resuspended in buffer for RNA isolation using an All-in-One purification kit (Norgen Biotek Corp., Thorold, ON, Canada). RNA was spectrophotometrically quantified (Nanodrop ND-1000 spectrophotometer; Nanodrop Technologies, Wilmington, DE, USA).
The miRNAs associated with Alix/Ago2 Co-IP were analyzed by reverse transcription-quantitative polymerase chain reaction ( $\mathrm{RT}-\mathrm{qPCR})$. cDNA obtained by reverse transcription of $200 \mathrm{ng}$ of RNA using the miScript Reverse Transcription kit was then subjected to RT-qPCR using the miScript SYBR Green PCR kit (both from Qiagen, Valencia, CA, USA) to detect and quantify the miRNAs of interest. Each reaction was run in triplicate using $4 \mathrm{ng}$ of cDNA. miRNAspecific primers to miR-24, miR-31, miR-125b, miR-99b, miR-221, miR-16 and miR-21 were used. As positive controls, RNU6B was used. RT-qPCR was accomplished using a 48-well StepOne Real-Time System (Applied Biosystems, Foster City, CA, USA). Negative cDNA controls (no cDNA) were cycled in parallel with each run. Data are expressed as normalized values of miRNA mean differences $\left(2^{\wedge}-\Delta \Delta \mathrm{Ct} \pm \mathrm{SD}\right)$.

miRNA transfer. The EV-mediated transfer of miRNAs to the HUVECs was evaluated by RT-qPCR performed on total RNA extracted from the HUVECs treated or not with HLSC-derived EVs and in the presence of actinomycin D (ActD; Sigma) as previously described (14). As a control, HUVECs were treated with $10 \mathrm{ng} / \mathrm{ml}$ of vascular endothelial growth factor (VEGF; PHC9394; Thermo Fisher Scientific, Waltham, MA, USA) in the absence or presence of ActD. Briefly, the HUVECs were treated for 30 min with ActD $(5 \mu \mathrm{g} / \mathrm{ml})$, to inhibit RNA transcription. After washing, the HUVECs were incubated with or without EVs $(100 \mu \mathrm{g} / \mathrm{ml})$ at different time-points. Following reverse transcription of the RNA (200 ng), the cDNA was subjected to RT-qPCR to quantify miRNAs as described above.

HLSC transfection. The shRNA plasmids were provided by Santa Cruz Biotechnology in a group of 3 to 5 lentiviral plasmids which act as carriers. Each plasmid was coding for a specific 19-25 (plus hairpins) shRNA designed for the reduction of gene expression. shRNA sequences corresponding to the Alix siRNA gene were provided as plasmid DNA ready for transfection. Following transfection, the cells stably expressing shRNA were isolated by puromycin selection.

The HLSCs were transfected using the Amaxa Nucleofector technology (Amaxa, Koeln, Germany). The cells were resuspended in a human MSC Nucleofector kit, according to the Amaxa guidelines. Briefly, $100 \mu 1$ of $4-5 \times 10^{5}$ cells added with $2 \mu \mathrm{g}$ cDNA were nucleofected with an Amaxa Nucleofector apparatus using U-23 (for high transfection efficiency) Nucleofector program. The cells were then transferred to 6-well plates containing $37^{\circ} \mathrm{C}$ pre-warmed culture medium. The positive control vector, pmaxGFP, which was rovided with the kit, was used to establish the best Nucleofactor program. The cells were observed under a Leica DMI3000 B microscope (Leica, Mannheim, Germany). As a negative control, cells in Nucleofector solution with DNA, but without application of the program and cells in Nucleofector solution without DNA with application of the program were used. Twenty-four hours following transfection, RNA was extracted from the cells in order to assess Alix gene expression decrease. Total RNA was extracted using a mirVana RNA isolation kit (Ambion, Life Technologies, Carlsbad, CA, USA). cDNA was obtained from total RNA using the High Capacity cDNA Reverse Transcription kit (Applied Biosystems). For each cDNA synthesis, $200 \mathrm{ng}$ mRNA, $2 \mu \mathrm{l}$ RT buffer, $0.8 \mu \mathrm{l}$ dNTP mixture, $2 \mu \mathrm{l}$ RT random primers, 
A

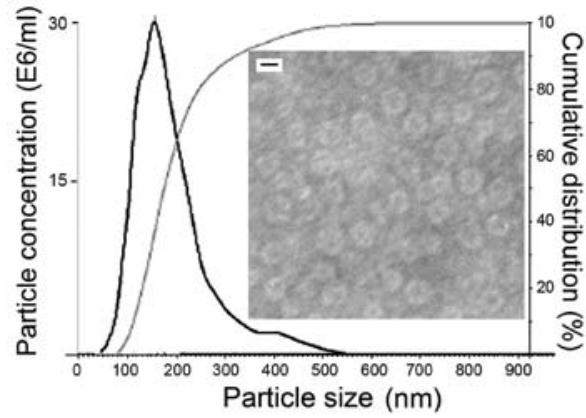

C

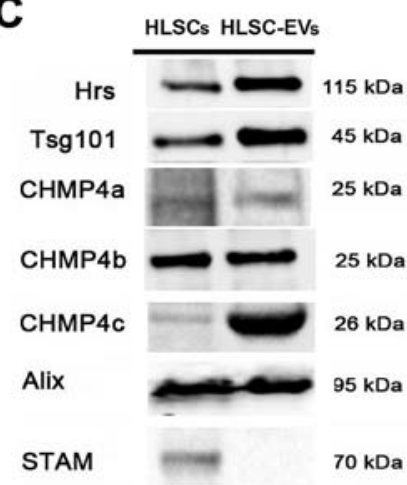

B

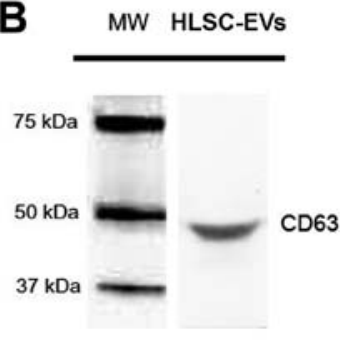

E

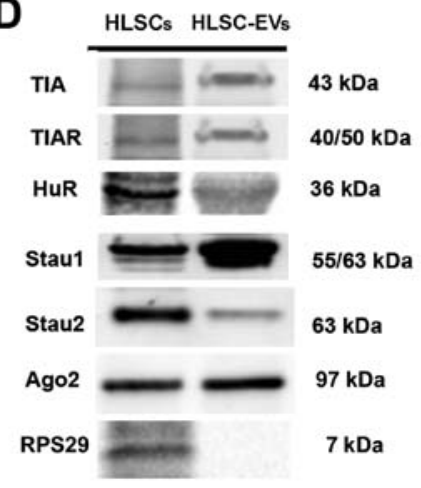

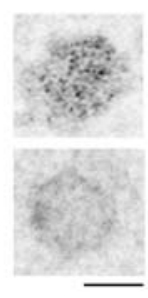

kDa

Figure 1. Characterization of human liver stem-like cell (HLSC)-derived extracellular vesicles (EVs; HLSC-EVs). (A) Representative NanoSight image of 100k g purified EVs; inset representative transmission electron microscopy image of purified EVs negatively stained with NanoVan (black line=100 nm). (B) Representative western blots of CD63 expression in HLSC-derived EVs. (C) Representative western blots of endosomal sorting complex required for transport (ESCRT) components (Tsg101, CHMP4 a,b,c, Alix and STAM) performed on HLSCs and HLSC-derived EVs. (D) Representative western blots showing the expression of ribonucleoproteins (TIA, TIAR, HuR, Stau1/2 and Ago2) and RPS29 in HLSCs and HLSC-derived EVs. Four experiments were performed with similar results. (E) Representative immunogold electron microscopy images showing staining for Ago2 (upper panel) and control where the primary antibody was omitted (lower panel). EVs were observed using a JEOL Jem-1010 electron microscope (black line, $100 \mathrm{~nm}$ ).

$1 \mu 1$ MultiScribe reverse transcriptase and $4.2 \mu 1$ nuclease-free water were used. A total of $100 \mathrm{nM}$ of each primer (Alix or $18 \mathrm{~S}$ ), were analyzed in the 48-well StepOne Real-Time System (Applied Biosystems). In parallel to each run, negative cDNA controls (no cDNA) were cycled. The following primers were used: human Alix F1, 5'-GACGCTCCTGAGATATTATGA TCAGA-3' and R1, 5'-ACACACAGCTCTTTTCATATCCTA AGC-3'; and human 18S F1, 5'-CCTGCCAGTAGCATATGC TTGTC-3' and R1, 5'-AGCCATTCGCAGTTTCACTGTA-3'.

The decrease in Alix protein expression was evaluated following puromycine selection $(250 \mathrm{ng} / \mathrm{ml})$. Alix knockdown was evaluated in the HLSCs and the HLSC-derived EVs by western blot analysis. Variations in the amount and profiles of the released EVs were evaluated by NanoSight analysis.

Statistical analysis. The Kolmogorov-Smirnov test was used for the evaluation of the normality of the distribution of data obtained by FACS analysis. The SigmaPlot11.0 software was used for statistical analysis and the Student's t-test was performed to evaluate the differences between the different groups. Data are expressed as the means $\pm \mathrm{SD}$. A value of $\mathrm{P}<0.05$ was considered to indicate a statistically significant difference.

\section{Results}

ESCRT marker expression in EVs released from HLSCs. The $100 \mathrm{k} g$ purified fraction of the EVs revealed (by NanoSight) a mean size of $164 \pm 30 \mathrm{~nm}$ (Fig. 1A). Using transmission elec- tron microscopy, the negatively stained EVs exhibited a round shape with a dense core. Using electron microscopy, the size of the fixed EVs was found to be approximately $100 \mathrm{~nm}$, smaller than that observed with NanoSight, which was influenced by the temperature.

FACS analysis revealed that the EVs expressed several surface molecules of HLSCs, such as CD29, CD44, human leukocyte antigen (HLA) I and integrin $\alpha 5$ (data not shown). Moreover, the results of western blot analysis revealed that the EVs expressed CD63 (Fig. 1B). The EVs were obtained after $16 \mathrm{~h}$ of starvation and the amount of EVs produced per single cell was $1321 \pm 256$.

Western blot analysis revealed that the EVs expressed, as did the cells of origin, several ESCRT proteins, including Hrs, Tsg101, CHMP4a, CHMP4b, CHMP4c and Alix (Fig. 1C). When compared with the cells, the expression of Hrs, Tsg101 and CHMP4c was increased in the EVs. By contrast, STAM was detectable only in the cells and not in the EVs. The positivity for ESCRT components in the EVs suggests their transfer from HLSCs during vesiculation.

Moreover, the EVs expressed TIA-1, TIAR and HuR, which are multifunctional ribonucleoproteins known to be expressed in nuclei and stress granules (37) (Fig. 1D). In addition, they expressed Stau 1 and 2 proteins, which are involved in the transport and stability of mRNAs (17), as well as Ago2, a protein of the Argonaute family, which is involved in the transport and processing of miRNAs (18) (Fig. 1D). The expression of Ago2 was confirmed by immunogold electron microscopy (Fig. 1E). 
A

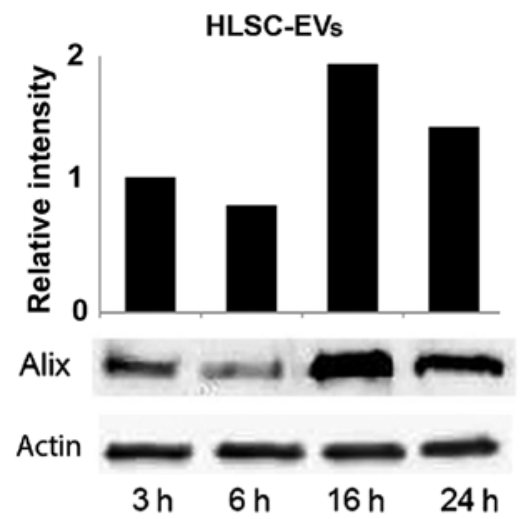

B

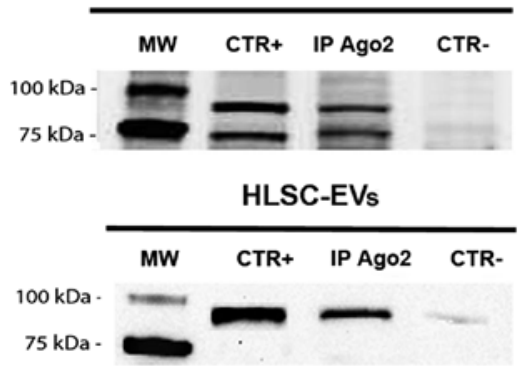

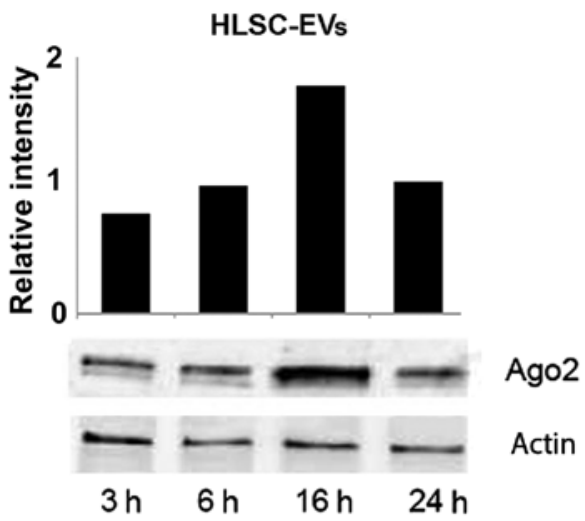

HLSCs

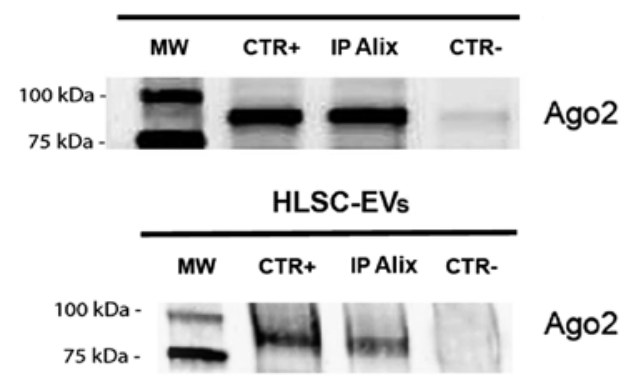

Figure 2. Alix and Ago2 co-immunoprecipitation (Co-IP). (A) Representative western blots and densitometric analysis of Alix and Ago2 expression in human liver stem-like cell (HLSC)-derived extracellular vesicles (EVs; HLSC-EVs) at 3, 6, 16, $24 \mathrm{~h}$ of cell starvation. (B) Representative Co-IP experiments performed on HLSCs (top panel) and HLSC-derived EVs (bottom panel) revealed interaction between Alix and Ago2. The immune complexes were formed by preincubation with anti-Ago2 (IP Ago2) and revealed with Alix antibody (left panels), or by pre-incubation with anti-Alix (IP Alix panels) and revealed with Ago2 antibody (right). For IP-positive control (CTR+), to evaluate the effective immunoprecipitation, the membranes were blotted with an antibody that recognises the same immunoprecipitated antigen. As a negative control (CTR-) non-immune IgG were used. Six experiments were performed with similar results.
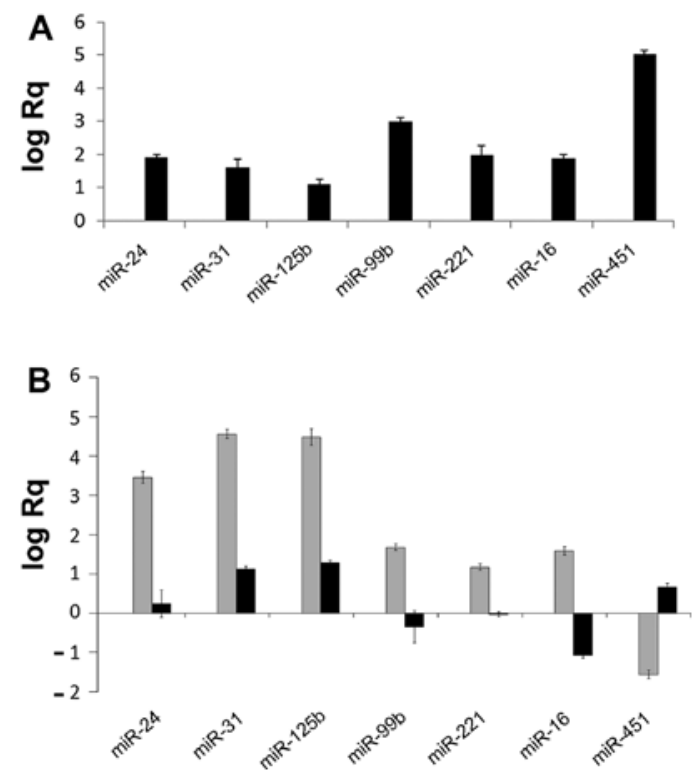

Figure 3. miRNA expression in human liver stem-like cell (HLSC)-derived extracellular vesicles (EVs; HLSC-EVs). (A) The expression of miR24, miR31, miR125b, miR99b, miR221, miR16 and miR451 was evaluated by RT-qPCR in HLSC-derived EVs with respect to HLSCs. Data are expressed as log of relative quantification (Rq), normalized to RNU6B and to one for HLSC. These miRNAs were enriched in EVs in respect to their relative HLSC. Data are the means \pm SD of 4 experiments. (B) The expression of miR24, miR31, miR125b, miR99b, miR221, miR16 and miR451 was evaluated by RT-qPCR in HLSCderived EVs immunoprecipitated with Ago2 (gray bars) and in HLSC-derived EVs immunoprecipitated with Alix (black bars) with respect to 100k g purified EVs. Data are expressed as the log of Rq, normalized to RNU6B and to one for $100 \mathrm{~kg}$ purified EVs. Data are the means \pm SD of 4 experiments.
The presence within the HLSC-derived EVs of selected ribonucleoproteins suggests a role in mRNA and miRNA storage.

We have previously demonstrated an enrichment of miRNAs within HLSC-derived EVs (14). Therefore, in this study, we focused on the interaction between Alix, one of the main expressed components of the ESCRT family in EVs, and Ago2 by performing Co-IP experiments.

Alix and Ago 2 interaction in HLSC-derived EVs. In kinetic experiments, we found that the expression of Alix and Ago2 in the EVs was highest after $16 \mathrm{~h}$ of HLSC starvation (Fig. 2A). As shown in Fig. 2B, an interaction between Alix and Ago2 was observed in the immunoprecipitates of the HLSCs and the EVs. We therefore investigated whether the Alix/Ago2 complex was involved in the enrichment of miRNAs within the HLSCderived EVs.

miRNA association with the Alix/Ago2 complex. On the basis of previous experiments that identified miRNAs with antitumor or regenerative activity in EVs derived from HLSCs $(23,38)$, we evaluated the enrichment of these miRNAs within the EVs. Fig. 3A shows the log-relative quantification of miR-24, miR-31, miR-125b, miR-99b, miR-221, miR-16 and miR-451 in the EVs with respect to the HLSCs evaluated by RT-qPCR. These results suggest that during vesiculation, selected miRNAs are compartmentalized within EVs.

Similarly, miRNA enrichment was observed in the Alix/Ago2 immunoprecipitate complex (Fig. 3B), suggesting 


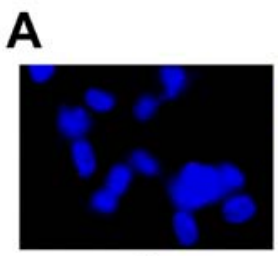

0

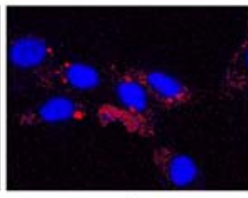

1

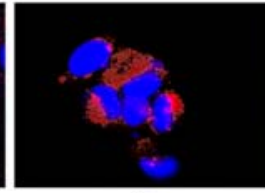

3

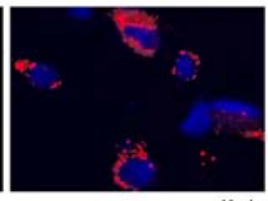

6

(h)
B

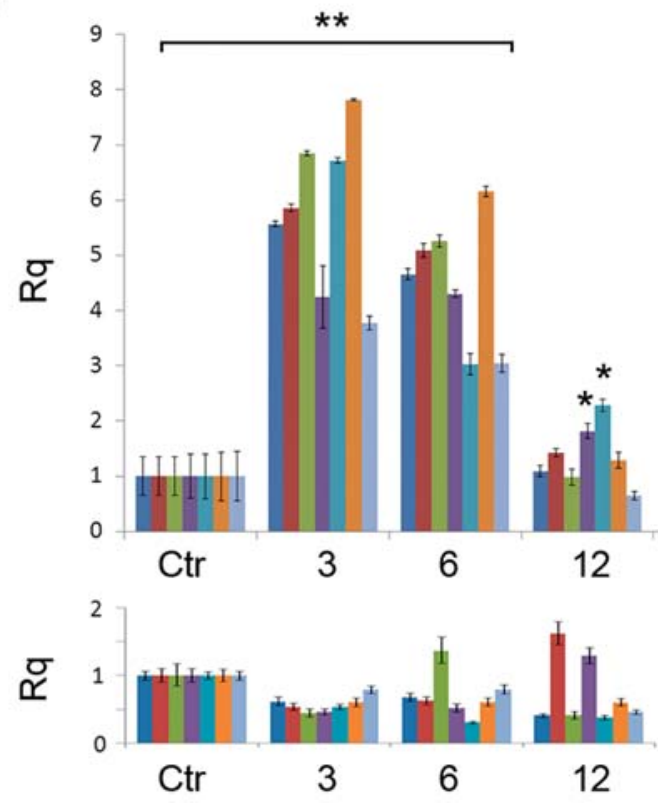

$\square \operatorname{miR}-24$

miR-31

miR-125b

a miR-99b

miR-221

a miR-16

- miR-451 (h)

(h)

Figure 4. Extracellular vesicle (EV) internalization and miRNA transfer in human umbilical cord vein-derived endothelial cells (HUVECs). (A) Representative image of internalization of PKH26-labeled human liver stem-like cell-derived EVs into HUVECs detected by confocal microscopy at different time-points. Three experiments were performed with similar results. (B, upper panel) RT-qPCR performed on HUVEC RNA treated with actinomycin D (ActD), in order to block RNA transcription, and incubated with HLSC-derived EVs at different time-points (3, 6 and $12 \mathrm{~h}$ ). HUVECs without EV stimulation at time 0 were used as control (Ctr) and set to one. (B lower panel) RT-qPCR performed on HUVEC RNA treated with ActD, in order to block RNA transcription, and incubated with vehicle alone at different times $(3,6$ and $12 \mathrm{~h})$. Data are expressed as relative quantification $(\mathrm{Rq})$, normalized to RNU6B. Data are the means \pm SD of 4 experiments. ${ }^{*} \mathrm{P}<0.05 ;{ }^{* *} \mathrm{P}<0.001$ vs. control (Ctr).

that the Alix/Ago2 complex was instrumental in miRNA accumulation within EVs.

To evaluate whether EVs are instrumental in transferring miRNAs to target cells, we used HUVECs as recipient cells. The extent of EV incorporation into the HUVECs was evaluated by FACS analysis (data not shown) and confocal microscopy (Fig. 4A). The PKH26-labeled HLSC-derived EVs were already detectable in the HUVECs following $1 \mathrm{~h}$ of incubation, with a maximum internalization after 3-6 h (Fig. 4A). RT-qPCR was performed on RNA from the HUVECs treated or not with HLSC-derived EVs in the presence of ActD in order to block RNA transcription. The effective block of RNA transcription by ActD was evaluated by monitoring miR-126 and miR-31 expression in the HUVECs stimulated with VEGF $(10 \mu \mathrm{g} / \mathrm{ml})$. The enhanced expression of these miRNAs was inhibited by ActD (data not shown). As shown in Fig. 4B, the accumulation of miRNAs in the HUVECs treated with EVs peaked after $3 \mathrm{~h}$ to progressively decrease at $12 \mathrm{~h}$. miRNA expression in the unstimulated HUVECs did not significantly vary with time.

Alix knockdown experiments were performed by plasmidic electroporation in HLSCs of shRNA against Alix to assess its effects on the release of EVs and miRNA content. The transfection efficiency evaluated as the green fluorescence protein (GFP) signal ranged between $80-90 \%$ in the HLSCs after $24 \mathrm{~h}$. At this time, a significant decrease in the Alix mRNA levels was detected by RT-qPCR. Moreover, the decrease in Alix protein expression was detected up to 2 weeks following selection with puromycin (Fig. 5A). Decreased Alix expression was also observed in the EVs derived from the HLSCs in which Alix had been knocked down. Moreover, the expression of Ago2 was significantly decreased in the EVs (Fig. 5B), but not in the HLSCs (data not shown), suggesting that Alix is required for Ago2 localization within EVs. As shown in Fig. 5C, Alix knockdown in the HLSCs significantly reduced miRNA compartmentalization in the EVs, confirming a critical role of the Alix/Ago2 complex in driving miRNAs within EVs. The reduction in the EV miRNA content resulted in a significant decrease in miRNA transfer to the HUVECs (Fig. 6). By contrast, Alix knockdown did not influence the amount of vesicles produced by the HLSCs as detected by NanoSight (wild-type HLSCs, $3.07 \pm 1.5 \times 10^{8}$ particles $/ \mathrm{ml}$; knockdown HLSCs, $3.68 \pm 1.5 \times 10^{8}$ particles $/ \mathrm{ml}$ ) suggesting that Alix was instrumental in the composition of EVs rather than in their secretion. 


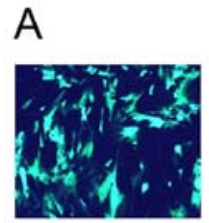

GFP
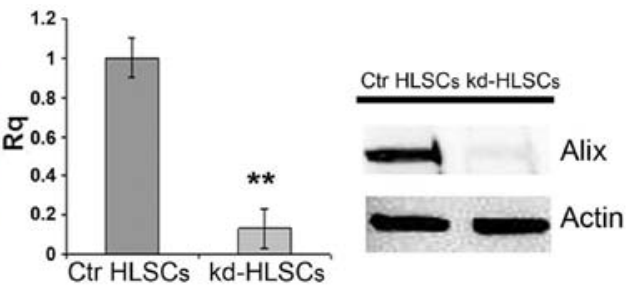

B
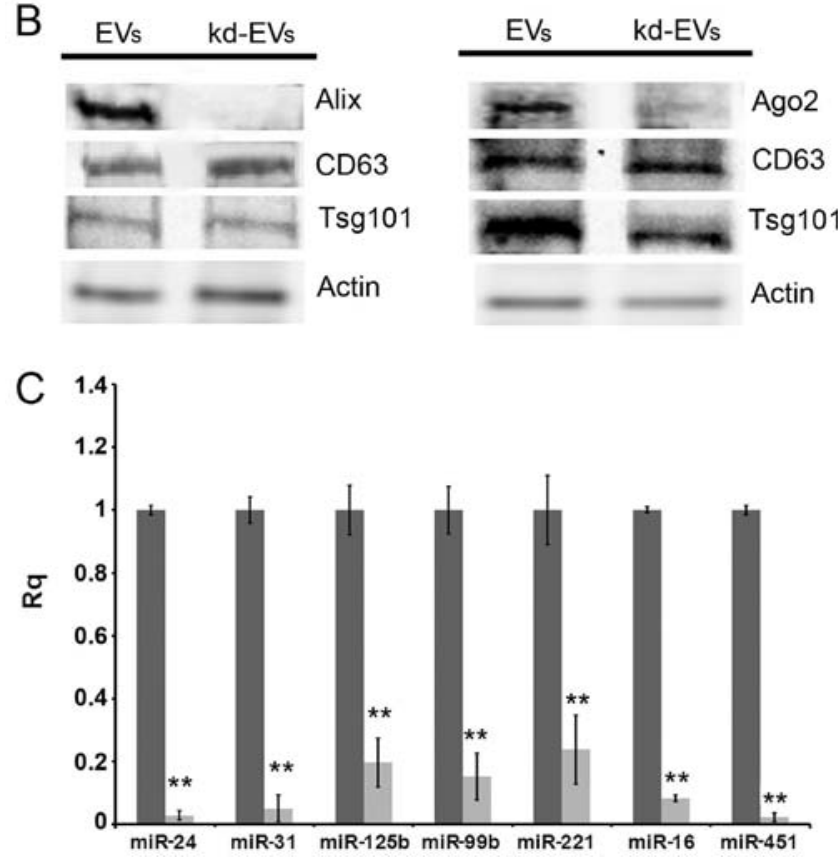

Figure 5. Effects of Alix knockdown in human liver stem-like cells (HLSCs). (A) Representative image of HLSCs transfected with the positive control vector, pmaxGFP, $24 \mathrm{~h}$ after transfection (original magnification, x100); Alix mRNA expression in HLSCs in which Alix was knocked down (kd-HLSCs) with respect to normal HLSCs (Ctr HLSCs) set to one, was evaluated by RT-qPCR. Data are the means \pm SD of 3 experiments expressed as relative quantification $(\mathrm{Rq})$, normalized to $18 \mathrm{~S}$. Representative western blots of Alix protein expression in kd-HLSCs with respect to Ctr HLSCs. Equal loading was confirmed by stripping the immunoblot and reprobing it for actin. Three different experiments were performed with similar results. (B) Representative western blots of Alix and Ago2 protein expression in extracellular vesicles (EVs) released from normal HLSCs (EVs) and in EVs released from HLSCs in which Alix was knocked down (kd-EVs). Equal loading was confirmed by stripping the immunoblot and reprobing it for CD63 and Tsg101. Three different experiments were performed with similar results. (C) The expression of miR-24, miR-31, miR-125b, miR-99b, miR-221, miR-16 and miR-451 was evaluated by RT-qPCR in EVs derived from HLSCs in which Alix was knocked down (gray bars) with respect to EVs derived from normal HLSCs (black bars) set to one. Data are expressed as Rq, normalized to RNU6B. Data are the means \pm SD of 4 experiments. ${ }^{* *} \mathrm{P}<0.001$ vs. HLSC-derived EVs.

\section{Discussion}

The results of the present study demonstrated that the EVs released from the HLSCs are enriched in proteins involved in ESCRT machinery, including Alix and miRNAs. Moreover, they contain several RNA-binding proteins, including Ago2, a component of the Argonaute family known to be involved in miRNA transport and processing. Co-IP experiments demonstrated that Alix binds to Ago2 and miRNAs, suggesting that Alix plays a key role in the miRNA enrichment during the biogenesis of EVs. We found that Alix was involved in the molecular composition of vesicles released from HLSCs, but

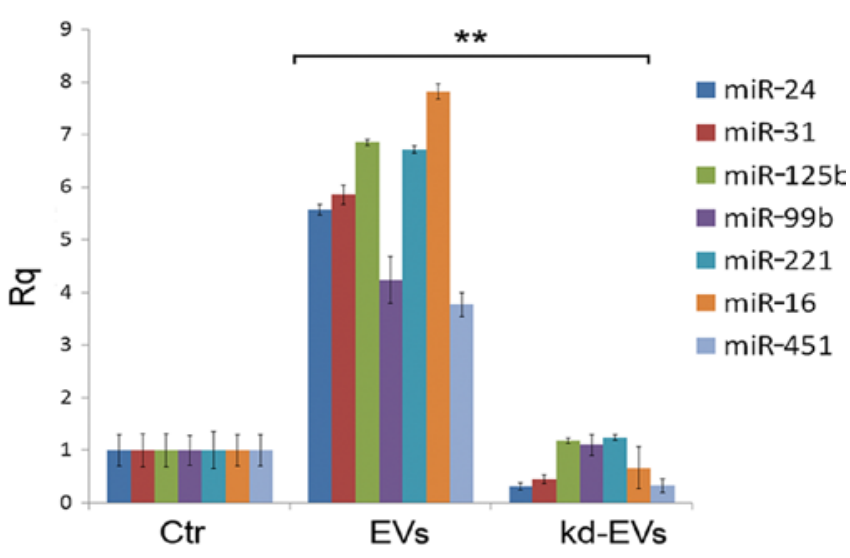

Figure 6. Effect of Alix knockdown on extracellular vesicle (EV)-mediated transfer of miRNAs to human umbilical cord vein-derived endothelial cells (HUVECs). RT-qPCR performed on RNA extracted from HUVECs treated with actinomycin D (ActD) and incubated with human liver stem-like cell (HLSC)-derived EVs (EV) or EVs derived from HLSCs in which Alix was knocked down (kd-EVs) for $3 \mathrm{~h}$. HUVECs without EV stimulation were used as controls (Ctr) and set to one. Data are expressed as relative quantification $(\mathrm{Rq})$, normalized to RNU6B. Data are the means \pm SD of 4 experiments. ${ }^{* *} \mathrm{P}<0.001$ kd-EVs vs. EVs.

not in their secretion. In fact, Alix knockdown did not influence the number of EVs released by the HLSCs, but it significantly decreased miRNA expression in the EVs and, consequently, their transfer to the endothelium.

EVs, important mediators of intercellular communication, have also recently emerged as new diagnostic (38) and therapeutic (39) tools. EVs have been shown to be involved in immune modulation (40), tumor biology (41) and tissue regeneration (42). The mechanisms of action of EVs are associated with their ability to directly stimulate target cells and/or to transfer proteins, biologically active lipids and nucleic acids. In particular, the transfer of transcription factors, mRNAs and non-coding RNAs has been shown to induce epigenetic modifications in recipient cells, leading to changes in cell function and phenotype (43). The understanding of the mechanisms involved in the biogenesis (6) and the loading of EV cargo is crucial, not only for the definition of their physiological role, but also for their possible engineering and exploitation in therapy $(42,44)$.

The compartment of biologically active molecules within EVs is modified by environmental and metabolic conditions, suggesting a tightly regulated mechanism which remains to be elucidated.

Alix has been implicated in the sorting of exosomes from infected and MCF7 cells during vesiculation (23-25). ESCRT machinery has been shown to participate in processes of vesiculation and cargo sorting, including multivesicular body biogenesis and the release of enveloped viruses (45-47). In the present study, we found that ESCRT proteins were expressed in HLSCs and in the released EVs, suggesting a role in EV formation. Alix, an accessory protein of the ESCRT complex, has multifunctional activities due to its domains, which provide multiple protein-binding sites $(24,28-33)$. In particular, it has been demonstrated that Alix is involved in endocytic membrane trafficking and cytoskeletal remodeling $(27,29,33,48)$. The N-terminal 'Brol domain' allows endosomal localization, whereas the $\mathrm{C}$-terminal region may interact with proteins that connect Alix with different cellular processes (28-33). Several 
viruses exploit Alix in order to bud from infected cells. An interaction between the nucleocapsid (NC) domain of HIV and the Brol domain of Alix has been demonstrated (32). In that study, it was suggested that RNA may serve as a bridge for Brol-NC interactions (32). Of note, Shen et al established a parallelism between EV biogenesis and retrovirus budding, suggesting that plasma membrane anchors may target highly oligomeric cytoplasmic proteins to EVs (49). They also demonstrated that higher-order oligomerization was the main determinant of HIV Gag budding/exosomal sorting $(50,51)$.

It has been suggested that the enrichment of extracellular RNA within EVs depends on its association with RNA-binding proteins $(14,52)$. We found that the EVs released from the HLSCs contained several RNA-binding proteins including Ago2, which is involved in miRNA transport and processing (18). Herein, we suggest that Alix, by forming a complex with Ago2, drives it within EVs together with the associated miRNAs. The observation that Alix knockdown in HLSCs prevented the accumulation of Ago2 and miRNAs in EVs, as well as the consequent transfer of miRNAs to target cells, further supports the relevance of this protein in modulating the composition of EVs.

In the future, it would be interesting in the future to evaluate whether the role of Alix in the enrichment of miRNA species in EVs is a general mechanism involved in other cell types. In conclusion, the results of the present study suggest a novel function of Alix, demonstrating its involvement in the composition of EVs released from HLSCs.

\section{Acknowledgements}

The present study was supported by the Associazione Italiana per la Ricerca sul Cancro (AIRC) project IG 12890 and by the National Center For Advancing Translational Sciences of the National Institutes of Health under Award number UH2TR000880. The content is solely the responsibility of the authors and does not necessarily represent the official views of the National Institutes of Health.

\section{References}

1. Quesenberry PJ, Goldberg LR, Aliotta JM, Dooner MS, Pereira MG, Wen S and Camussi G: Cellular phenotype and extracellular vesicles: basic and clinical considerations. Stem Cells Dev 23: 1429-1436, 2014.

2. Ratajczak J, Wysoczynski M, Hayek F, Janowska-Wieczorek A and Ratajczak MZ: Membrane-derived microvesicles: important and underappreciated mediators of cell-to-cell communication. Leukemia 20: 1487-1495, 2006.

3. Théry C, Ostrowski M and Segura E: Membrane vesicles as conveyors of immune responses. Nat Rev Immunol 9: 581-593, 2009.

4. Lee TH, D'Asti E, Magnus N, Al-Nedawi K, Meehan B and Rak J: Microvesicles as mediators of intercellular communication in cancer - the emerging science of cellular 'debris'. Semin Immunopathol 33: 455-467, 2011.

5. Cocucci E, Racchetti G and Meldolesi J: Shedding microvesicles: artefacts no more. Trends Cell Biol 19: 43-51, 2009.

6. Colombo M, Raposo G and Théry C: Biogenesis, secretion, and intercellular interactions of exosomes and other extracellular vesicles. Annu Rev Cell Dev Biol 30: 255-289, 2014.

7. Gould SJ and Raposo G: As we wait: coping with an imperfect nomenclature for extracellular vesicles. J Extracell Vesicles 2: Feb 15, 2013 (Epub ahead of print). doi: 10.3402/jev.v2i0.20389.

8. Chen X, Liang H, Zhang J, Zen K and Zhang CY: Secreted microRNAs: a new form of intercellular communication. Trends Cell Biol 22: 125-132, 2012.
9. Wang K, Zhang S, Weber J, Baxter D and Galas DJ: Export of microRNAs and microRNA-protective protein by mammalian cells. Nucleic Acids Res 38: 7248-7259, 2010.

10. Gallo A, Tandon M, Alevizos I and Illei GG: The majority of microRNAs detectable in serum and saliva is concentrated in exosomes. PLoS One 7: e30679, 2012.

11. Ratajczak J, Miekus K, Kucia M, Zhang J, Reca R, Dvorak P and Ratajczak MZ: Embryonic stem cell-derived microvesicles reprogram hematopoietic progenitors: evidence for horizontal transfer of mRNA and protein delivery. Leukemia 20: 847-856, 2006.

12. Deregibus MC, Cantaluppi V, Calogero R, Lo Iacono M, Tetta C, Biancone L, Bruno S, Bussolati B and Camussi G: Endothelial progenitor cell derived microvesicles activate an angiogenic program in endothelial cells by a horizontal transfer of mRNA. Blood 110: 2440-2448, 2007.

13. Valadi H, Ekström K, Bossios A, Sjöstrand M, Lee JJ and Lötvall JO: Exosome-mediated transfer of mRNAs and microRNAs is a novel mechanism of genetic exchange between cells. Nat Cell Biol 9: 654-659, 2007.

14. Collino F, Deregibus MC, Bruno S, Sterpone L, Aghemo G, Viltono L, Tetta $\mathrm{C}$ and Camussi G: Microvesicles derived from adult human bone marrow and tissue specific mesenchymal stem cells shuttle selected pattern of miRNAs. PLoS One 5: e11803, 2010.

15. Aliotta JM, Sanchez-Guijo FM, Dooner GJ, Johnson KW, Dooner MS, Greer KA, Greer D, Pimentel J, Kolankiewicz LM, Puente $\mathrm{N}$, et al: Alteration of marrow cell gene expression, protein production, and engraftment into lung by lung-derived microvesicles: a novel mechanism for phenotype modulation. Stem Cells 25: 2245-2256, 2007.

16. Gibbings D, Leblanc P, Jay F, Pontier D, Michel F, Schwab Y, Alais S, Lagrange $\mathrm{T}$ and Voinnet $\mathrm{O}$ : Human prion protein binds Argonaute and promotes accumulation of microRNA effector complexes. Nat Struct Mol Biol 19: 517-524, S1, 2012.

17. Laver JD, Li X, Ancevicius K, Westwood JT, Smibert CA, Morris QD and Lipshitz HD: Genome-wide analysis of Staufenassociated mRNAs identifies secondary structures that confer target specificity. Nucleic Acids Res 41: 9438-9460, 2013.

18. Waris S, Wilce MC and Wilce JA: RNA recognition and stress granule formation by TIA proteins. Int J Mol Sci 15: 23377-23388, 2014.

19. Höck $\mathbf{J}$ and Meister G: The Argonaute protein family. Genome Biol 9: 210, 2008.

20. Herrera MB, Fonsato V, Bruno S, Grange C, Gilbo N, Romagnoli R, Tetta C and Camussi G: Human liver stem cells improve liver injury in a model of fulminant liver failure. Hepatology 57: 311-319, 2013.

21. Herrera MB, Fonsato V, Gatti S, Deregibus MC, Sordi A, Cantarella D, Calogero R, Bussolati B, Tetta C and Camussi G: Human liver stem cell-derived microvesicles accelerate hepatic regeneration in hepatectomized rats. J Cell Mol Med 14: 1605-1618, 2010.

22. Herrera MB, Bruno S, Buttiglieri S, Tetta C, Gatti S, Deregibus MC, Bussolati B and Camussi G: Isolation and characterization of a stem cell population from adult human liver. Stem Cells 24: 2840-2850, 2006.

23. Fonsato V, Collino F, Herrera MB, Cavallari C, Deregibus MC, Cisterna B, Bruno S, Romagnoli R, Salizzoni M, Tetta C and Camussi G: Human liver stem cell-derived microvesicles inhibit hepatoma growth in SCID mice by delivering antitumor microRNAs. Stem cells 30:1985-1998,2012.

24. Martin-Serrano J, Yarovoy A, Perez-Caballero D and Bieniasz PD: Divergent retroviral late-budding domains recruit vacuolar protein sorting factors by using alternative adaptor proteins. Proc Natl Acad Sci USA 100: 12414-12419, 2003.

25. Baietti MF, Zhang Z, Mortier E, Melchior A, Degeest G, Geeraerts A, Ivarsson Y, Depoortere F, Coomans C, Vermeiren E, et al: Syndecan-syntenin-ALIX regulates the biogenesis of exosomes. Nat Cell Biol 14: 677-685, 2012.

26. Roucourt B, Meeussen S, Bao J, Zimmermann P and David G: Heparanase activates the syndecan-syntenin-ALIX exosome pathway. Cell Res 25: 412-428, 2015.

27. Ghossoub R, Lembo F, Rubio A, Gaillard CB, Bouchet J, Vitale N, Slavík J, Machala M and Zimmermann P: Syntenin-ALIX exosome biogenesis and budding into multivesicular bodies are controlled by ARF6 and PLD2. Nat Commun 5: 3477, 2014.

28. Missotten M, Nichols A, Rieger K and Sadoul R: Alix, a novel mouse protein undergoing calcium-dependent interaction with the apoptosis-linked-gene 2 (ALG-2) protein. Cell Death Differ 6: 124-129, 1999. 
29. Odorizzi G, Katzmann DJ, Babst M, Audhya A and Emr SD Brol is an endosome-associated protein that functions in the MVB pathway in Saccharomyces cerevisiae. J Cell Sci 116: 1893-1903, 2003.

30. Schmidt MH, Hoeller D, Yu J, Furnari FB, Cavenee WK, Dikic I and Bögler O: Alix/AIP1 antagonizes epidermal growth factor receptor downregulation by the Cbl-SETA/CIN85 complex. Mol Cell Biol 24: 8981-8993, 2004.

31. Strack B, Calistri A, Craig S, Popova E and Göttlinger HG: AIP1/ ALIX is a binding partner for HIV-1 p6 and EIAV p9 functioning in virus budding. Cell 114: 689-699, 2003.

32. Sette P, Dussupt $V$ and Bouamr F: Identification of the HIV-1 NC binding interface in Alix Brol reveals a role for RNA. J Virol 86: 11608-11615, 2012.

33. Morita E, Sandrin V, Chung HY, Morham SG, Gygi SP Rodesch CK and Sundquist WI: Human ESCRT and ALIX proteins interact with proteins of the midbody and function in cytokinesis. EMBO J 26: 4215-4227, 2007.

34. Dragovic RA, Gardiner C, Brooks AS, Tannetta DS, Ferguson DJ, Hole P, Carr B, Redman CW, Harris AL, Dobson PJ, et al: Sizing and phenotyping of cellular vesicles using Nanoparticle Tracking Analysis. Nanomedicine 7: 780-788, 2011.

35. Lopatina T, Bruno S, Tetta C, Kalinina N, Porta M and Camussi G: Platelet-derived growth factor regulates the secretion of extracellular vesicles by adipose mesenchymal stem cells and enhances their angiogenic potential. Cell Commun Signal 12: 26, 2014.

36. Deregibus MC, Buttiglieri S, Russo S, Bussolati B and Camussi G: CD40-dependent activation of phosphatidylinositol 3-kinase/Akt pathway mediates endothelial cell survival and in vitro angiogenesis. J Biol Chem 278: 18008-18014, 2003.

37. Anderson P and Kedersha N: RNA granules. J Cell Biol 172: 803-808, 2006.

38. Lin J, Li J, Huang B, Liu J, Chen X, Chen XM, Xu YM, Huang LF and Wang XZ: Exosomes: novel biomarkers for clinical diagnosis. ScientificWorldJournal 2015: 657086, 2015.

39. Katoh M: Therapeutics targeting angiogenesis: genetics and epigenetics, extracellular miRNAs and signaling networks (Review). Int J Mol Med 32: 763-767, 2013.

40. Chaput $\mathrm{N}$ and Théry C: Exosomes: Immune properties and potential clinical implementations. Semin Immunopathol 33: 419-440, 2011.
41. Milane L, Singh A, Mattheolabakis G, Suresh M and Amiji MM: Exosome mediated communication within the tumor microenvironment. J Control Release 219: 278-294, 2015.

42. De Jong OG, Van Balkom BW, Schiffelers RM, Bouten CV and Verhaar MC: Extracellular vesicles: potential roles in regenerative medicine. Front Immunol 5: 608, 2014.

43. Quesenberry PJ, Aliotta J, Deregibus MC and Camussi G: Role of extracellular RNA-carrying vesicles in cell differentiation and reprogramming. Stem Cell Res Ther 6: 153, 2015.

44. Zhang Y, Wu XH, Luo CL, Zhang JM, He BC and Chen G: Interleukin-12-anchored exosomes increase cytotoxicity of $\mathrm{T}$ lymphocytes by reversing the JAK/STAT pathway impaired by tumor-derived exosomes. Int J Mol Med 25: 695-700, 2010.

45. Morita E: Differential requirements of mammalian ESCRTs in multivesicular body formation, virus budding and cell division. FEBS J 279: 1399-1406, 2012.

46. Roxrud I, Stenmark H and Malerød L: ESCRT \& Co. Biol Cell 102: 293-318, 2010.

47. Votteler $\mathbf{J}$ and Sundquist WI: Virus budding and the ESCRT pathway. Cell Host Microbe 14: 232-241, 2013.

48. Pan S, Wang R, Zhou X, He G, Koomen J, Kobayashi R, Sun L, Corvera J, Gallick GE and Kuang J: Involvement of the conserved adaptor protein Alix in actin cytoskeleton assembly. J Biol Chem 281: 34640-34650, 2006.

49. Shen B, Wu N, Yang JM and Gould SJ: Protein targeting to exosomes/microvesicles by plasma membrane anchors. J Biol Chem 286: 14383-14395, 2011.

50. Fang Y, Wu N, Gan X, Yan W, Morrell JC and Gould SJ: Higher-order oligomerization targets plasma membrane proteins and HIV gag to exosomes. PLoS Biol 5: e158, 2007.

51. Booth AM, Fang Y, Fallon JK, Yang JM, Hildreth JE and Gould SJ: Exosomes and HIV Gag bud from endosome-like domains of the T cell plasma membrane. J Cell Biol 172: 923-935, 2006.

52. Li L, Zhu D, Huang L, Zhang J, Bian Z, Chen X, Liu Y, Zhang CY and Zen K: Argonaute 2 complexes selectively protect the circulating microRNAs in cell-secreted microvesicles. PLoS One 7: e46957, 2012 